ManN, S. O. \& Oxford, A. E. (1954). J. gen. Microbiol. 11, 83-90.

\title{
Studies of some Presumptive Lactobacilli isolated from the Rumens of Young Calves
}

\author{
By S. O. MANN AND A. E. OXFORD \\ The Rowett Research Institute, Bucksburn, Aberdeenshire
}

SUMMARY: Ten out of thirteen isolates of Gram-positive rods from the rumens of young calves proved to be catalase-negative and were further studied by methods suitable for lactobacilli. Four isolates from two calves, one of which was aureomycinfed, proved to be Lactobacillus brevis. Three isolates from a very young calf not fed aureomycin were provisionally identified as an anaerobic variant of $\boldsymbol{L}$. lactis. The remaining three isolates obtained from an aureomycin-fed calf were all motile, homofermentative and mannitol-fermenting, and could not be identified with any known species of Lactobacillus; this organism produced a mixture of dextrorotatory and inactive lactic acid from glucose. The fermentation reactions of all the presumed lactobacilli isolated in this study were clear-cut and reproducible.

Mann, Masson \& Oxford (1954) fed aureomycin (40-60 mg.) once daily to calves from the 2 nd to the 15 th week of age, but on slaughter could detect the antibiotic only in the abomasum, never in the rumen. Furthermore, the only noteworthy bacteriological differences between the rumen microflora of aureomycin-fed and control calves were that the rumen of the youngest control calf contained a considerable population of anaerobic Gram-positive rods not found in two aureomycin fed calves of the same age, and that amylolytic streptococci were rather more plentiful in the rumens of older control calves than in those of aureomycin-fed animals.

This paper is devoted to a description of thirteen isolates of Gram-positive rods (including three anaerobes) obtained in the foregoing study; most of them seemed to be lactobacilli. The accompanying Gram-positive cocci (sixtynine isolates) and facultatively anaerobic Gram-negative rods (forty isolates) will be described later.

\section{METHODS}

Feeding of calves. This is fully described by Mann et al. (1954). In this connexion the relevant points are that fresh unpasteurized T.T. milk was fed up to the 6th week of age, and that the aureomycin was administered not in the milk but dissolved in an aqueous gruel.

Isolation of rumen bacteria in pure culture. Unless otherwise stated, all incubations were done at $38^{\circ}$. Bottle counts on rumen contents immediately after slaughter of the calf were carried out by the method of Heald, Krogh, Mann, Appleby, Masson \& Oxford (1953) devised for sheep rumen contents. The basal agar contained absolutely clear sterilized sheep rumen liquor since it was not practicable to prepare clear calf rumen liquor on the scale required. As mentioned by Mann et al. (1954) both cellobiose and mannitol were employed as carbohydrate substrates. These authors also listed the actual viable counts 
obtained, which were never very large, i.e. more than $10^{8} / \mathrm{g}$., save with the first (1 C) and last (3C) control calves, aged 32 and 86 days respectively. Pure culture isolations were made from all bottles which contained $c$. 10-50 clearly visible and well-separated colonies after 3 days' incubation at $37^{\circ}$. Except with the first control calf ( $1 \mathrm{C} ; 32$ days old) these proved mostly to consist of Gram-positive cocci or small Gram-negative rods. Cultures of Gram-positive rods were, however, also obtained from the rumens of each of the following three calves (out of a total of eight, excluding $1 \mathrm{C}$ ) : 1 A (aureomycin-fed, 33 days old); $2 \mathrm{~A}$ (aureomycin-fed, 54 days old) and $3 \mathrm{~A}^{1}$ (aureomycin-fed, 96 days old). It should be emphasized that the initial isolation was not made from an agar medium especially devised for the isolation of lactobacilli. Consequently, it is unlikely that any exacting milk lactobacilli, the growth requirements of which might not be met by rumen liquor, would be detected in our counts, unless of course they were making use of growth factors synthesized by other bacteria forming colonies in the same bottle.

The cultures of rumen Gram-positive rods were purified by plating from glucose yeast-extract peptone broth on to agars of the same composition, which were incubated both aerobically and anaerobically. It was found that Robertson's cooked meat medium was not suitable for the maintenance of stock cultures, which were therefore kept in the freeze-dried state wherever possible.

Bacteriological methods. These followed those of Rogosa, Wiseman, Mitchell, Disraely \& Beaman (1953) very closely, since we were primarily concerned with the fermentation reactions of our organisms, and these authors had claimed almost absolute reproducibility under their conditions of test. Gas production from glucose (distinction between homofermentative and heterofermentative lactobacilli) was observed in the complex tomato juice agar stab cultures recommended by these authors. Fermentation tests were carried out in duplicate exactly as recommended, with washed suspensions from $24 \mathrm{hr}$. tomato-broth cultures as inocula ( $48 \mathrm{hr}$. cultures for the anaerobes). The whole series of fermentation tests was repeated several weeks later in order to test their reproducibility.

\section{RESULTS}

Origin of cultures of rumen Gram-positive rods. Such cultures were isolated from both cellobiose- and mannitol-containing bottles used in the count. Table 1 shows the several highest dilutions of rumen contents at which Grampositive rods were isolatable. They were always in evidence in direct rumen smears, particularly from the younger calves. Of the thirteen isolates listed in Table 1 only nos. 1-10 were catalase-negative and showed no ability to reduce nitrate. Isolates 11,12 and 13 (from the oldest calf) were catalasepositive and reduced nitrate; although they grew quite well at $38^{\circ}$ under anaerobic conditions and did not seem to form spores, obviously they did not belong to the genus Lactobacillus as usually defined, and were therefore not studied further.

On the basis of morphology and fermentation reactions the first ten isolates were found to fall into three groups only, all isolates in each group being 
virtually identical. These three groups were clearly differentiated from each other and are regarded as representing three separate and distinct rumen organisms, namely organism 123 (comprising isolates 1-3), organism 678 (comprising isolates 6-8) and organism 45910 (comprising isolates 4, 5, 9 and 10) respectively. It will be seen from Table 1 that the first and second organisms were each derived from one calf only (although in the second instance both mannitol and cellobiose bottles provided isolates), whereas the last organism (45910) comprised two isolates from each of two calves, one of which was fed aureomycin and the other not.

\section{Table 1. Origins of cultures of rumen Gram-positive rods}

\begin{tabular}{|c|c|c|c|c|}
\hline $\begin{array}{l}\text { Calf } \\
\text { no. }\end{array}$ & $\begin{array}{l}\text { Age of } \\
\text { calf } \\
\text { (days) }\end{array}$ & $\begin{array}{c}\text { Isolate } \\
\text { no. }\end{array}$ & $\begin{array}{c}\text { Highest dilution } \\
\text { and carbohydrate } \\
\text { used in } \\
\text { bottle count }\end{array}$ & $\begin{array}{l}\text { Whether Gram- } \\
\text { positive cocci } \\
\text { also isolated from } \\
\text { same bottle }\end{array}$ \\
\hline $1 \mathrm{C}$ & 32 & $\begin{array}{l}\mathbf{1} \\
\mathbf{2} \\
\mathbf{3} \\
\mathbf{4} \\
\mathbf{5}\end{array}$ & $\left.\begin{array}{l}c: \mathrm{CL} \\
a: \mathrm{CLV} \\
a: \mathrm{CLV}\end{array}\right\}$ & $\begin{array}{l}\text { No } \\
\text { No } \\
\text { Yes }\end{array}$ \\
\hline $1 \mathrm{~A}$ & 33 & $\begin{array}{l}6 \\
7 \\
8\end{array}$ & $\left.\begin{array}{l}b: \mathbf{C} \\
b: \mathbf{M} \\
b: \mathbf{M}\end{array}\right\}$ & $\begin{array}{l}\text { Yes } \\
\text { Yes }\end{array}$ \\
\hline $\mathbf{2 A}$ & 54 & $\begin{array}{r}9 \\
10\end{array}$ & $\left.\begin{array}{l}c: \mathbf{C} \\
c: \mathbf{C}\end{array}\right\}$ & Yes \\
\hline $\mathbf{3} \mathbf{A}^{\mathbf{1}}$ & 96 & $\begin{array}{l}11 \\
12 \\
13\end{array}$ & $\left.\begin{array}{l}a: \mathrm{M} \\
a: \mathbf{M} \\
a: \mathbf{M}\end{array}\right\}$ & Yes \\
\hline
\end{tabular}

Calves: $A$ or $A^{1}$, aureomycin-fed; $C$, control, not fed aureomycin (see Mann, Masson \& Oxford, 1954).

Dilutions of rumen contents : $a=1 / 10,000 ; b=1 / 500,000 ; c=1 / 3,000,000$.

Carbohydrates: CL, cellobiose; CLV, cellobiose + crystal violet $(0 \cdot 0005 \%)$; M, mannitol.

\section{Properties of organism 123}

This organism was obtained only from the rumen of the first control calf. It formed colonies only in the cellobiose nutrient agar used for the viable bacterial count, not in the corresponding medium containing mannitol. It was an obligate anaerobe and in the bottle count grew below the surface of the agar to the exclusion, at the higher dilutions, of all other visible colonies either above or below the line of demarcation of the growth. A very similar picture was presented when the organism was grown in pure culture in glucose tomatoextract agar. In liquid medium, likewise, there was no growth in any instance unless the tube was incubated in an anaerobic jar. The organism seemed to have no special $\mathrm{CO}_{2}$ requirement. On blood agar or nutrient agar plates, incubated anaerobically, the organism formed rough colonies, $1.5 \mathrm{~mm}$. in diameter, with undulating edge. The organism grew well at $45^{\circ}$ but not at $15^{\circ}$. None of the three isolates was aureomycin resistant.

Morphology. Films made from fluid cultures of organism 123, at all ages, and stained by Gram's method showed short and long (unbranched) filamentous 
rods, often highly curled and several hundred microns in length. These long filaments were not seen in direct smears of rumen contents. The staining was uneven and showed granulation. Gram-negative elements were also in evidence in older cultures, although the predominant appearance was always of Grampositive rods and filaments. Spores were never observed; the organism was non-motile.

Biochemical reactions. Organism 123 was weakly and inconsistently heterofermentative by the test of Rogosa et al. (1953). It produced acid followed by coagulation in litmus milk in 4 days at $38^{\circ}$.

The following carbohydrates were fermented with production of an acid $\mathrm{pH}$ value (numerical value in brackets) in each instance : aesculin $(4 \cdot 3)$; cellobiose $(4 \cdot 2)$; dextrin $(4 \cdot 6)$; fructose $(4 \cdot 1)$; galactose $(4 \cdot 3)$; glucose $(4 \cdot 2)$; lactose $(4 \cdot 2)$; maltose $(4 \cdot 2)$; mannose $(4 \cdot 2)$; melibiose $(4 \cdot 5)$; raffinose $(4 \cdot 3)$; salicin $(4 \cdot 4)$; sucrose $(4 \cdot 3)$; trehalose $(4 \cdot 2)$. The following compounds were not fermented, i.e. the $\mathrm{pH}$ value of the medium remained constant or fell only one or two tenths of a unit from the original value $(5 \cdot 9)$ even when growth occurred: arabinose, adonitol, dulcitol, glycerol, inositol, inulin, mannitol, melezitose, rhamnose, sorbitol, starch, xylose. All three isolates of organism 123 behaved exactly similarly in fermentation tests, which, when repeated several weeks later, gave the identical picture shown above.

In a fermentation of $500 \mathrm{ml}$. of a $2 \%$ glucose tomato-extract broth, the $\mathrm{pH}$ value fell to $4 \cdot 1$ in a few days at $37^{\circ}$, giving a copious white bacterial deposit (c. $0 \cdot 3 \mathrm{~g}$. when dried), with an absolutely clear supernatant from which the zinc salt of laevorotatory lactic acid was easily isolated by the standard method. In our opinion Briggs (1953) overestimates the difficulty of carrying out this important test, the sign of rotation of the lactic acid being of considerable diagnostic value. It is important, however, actually to determine the percentage of lactate in the crude zinc salt so isolated, by a specific method such as that of Barker \& Summerson (1941), since malic acid, which also can exhibit optical activity, is a possible fermentation product with lactobacilli. Even so, the total time consumed in these procedures is not great; about two working days. No zinc lactate could be isolated from the unsown medium when put through the same process.

Organism 123 did not hydrolyse hippurate or liquefy gelatin. It did not produce a soluble haemolysin.

Pathogenicity. Non-pathogenic to mice when injected intramuscularly or intraperitoneally; no local lesions.

\section{Properties of organism 678}

This was a facultative anaerobe; it yielded translucent colonies 0.75-1.0 mm. diameter, with raised centre and undulating edge with wavy projections after 2 days' incubation on nutrient agar. When grown in poured glucose agar deeps incubated aerobically, smooth colonies were formed at all levels. The organism grew profusely in glucose tomato-extract medium with good turbidity and sediment. Motility was observed after $16 \mathrm{hr}$. with all three isolates; this observation was repeated many times. The motility, although sluggish, was 
unmistakable even in a liquid culture which filled a $500 \mathrm{ml}$. conical flask with a narrow neck. None of the isolates (6-8) was aureomycin-resistant. The organism grew well at $45^{\circ}$ but not at all at $15^{\circ}$.

Morphology. Films made from fluid cultures and stained by Gram's method showed straight and curved Gram-positive rods with rounded ends ranging from 2 to $7 \mu$. in length, diameter $0.75 \mu$., together with some longer filaments up to $48 \mu$. long. Staining was variable and some Gram-negative elements were observed in cultures of any age. Spores were never seen.

Biochemical reactions. The organism was homofermentative by the test of Rogosa et al. (1953); it acidified and coagulated litmus milk. The following carbohydrates were fermented with production of an acid $\mathrm{pH}$ value (numerical value in brackets) in each instance: aesculin (4.3), cellobiose $(4 \cdot 0)$, dextrin $(4 \cdot 6)$, fructose $(3 \cdot 9)$, galactose $(4 \cdot 1)$, glucose $(3 \cdot 9)$, lactose $(4 \cdot 1)$, maltose $(4 \cdot 0)$, mannitol $(4 \cdot 0)$, mannose $(4 \cdot 1)$, raffinose $(3 \cdot 9)$, salicin $(4 \cdot 1)$, sorbitol $(4 \cdot 1)$, sucrose $(4 \cdot 0)$, trehalose $(3 \cdot 8)$, xylose $(5 \cdot 0-5 \cdot 4)$, melibiose $(4 \cdot 1)$. The following compounds were not fermented: arabinose, adonitol, dulcitol, glycerol, inositol, inulin, melezitose, rhamnose and starch. The organism thus has wider fermentative powers than the anaerobic organism 123, although the fermentation of xylose was weak. Repetition of the whole series of fermentation tests yielded exactly the same results.

In a fermentation of $500 \mathrm{ml}$. of a $2 \%$ glucose tomato-extract broth the $\mathrm{pH}$ value fell to 4.0 in 6 days at $37^{\circ}$, giving a much greyer bacterial deposit (after centrifuging) than with the anaerobic organism 123, although the yield of dried cells was only slightly less. Inactive and dextrorotatory lactic acid were the only fermentation products. No volatile acid was produced.

Organism 678 did not hydrolyse hippurate or liquefy gelatin. It produced distinct $\alpha$-haemolysis of sheep red cells when grown on blood-agar plates.

Pathogenicity. Non-pathogenic to mice, as organism 123.

\section{Properties of organism 45910}

This was a facultative anaerobe, but it did not grow as profusely in liquid media or on nutrient or (particularly) blood agar as did organism 678. The colonies were $0 \cdot 5-1 \cdot 0 \mathrm{~mm}$. diameter, smooth, translucent with entire edge and raised centre. All four isolates (4, 5, 9 and 10) grew better under microaerophilic conditions, or in an atmosphere containing $10 \% \mathrm{CO}_{2}$. In liquid media growth was chiefly granular with some turbidity but no pellicle. The organism was non-motile. None of the isolates was aureomycin-resistant. The organism grew well at $45^{\circ}$ but not at all at $15^{\circ}$.

Morphology. This was very similar indeed to that of organism 678; the rods in general were rather thicker, of diameter $1.0 \mu$.

Biochemical reactions. Organism 45910 was strongly heterofermentative (distinction from organisms 123 and 678). It acidified and coagulated litmus milk. The following carbohydrates were fermented with production of an acid $\mathrm{pH}$ value (numerical value in brackets) in each instance: arabinose (4.0), aesculin $(4 \cdot 8)$, fructose $(4 \cdot 7)$, galactose $(4 \cdot 4)$, glucose $(4 \cdot 4)$, lactose $(4 \cdot 5)$, maltose $(4 \cdot 5)$, melibiose $(4 \cdot 5)$, raffinose $(4 \cdot 6)$, salicin $(4 \cdot 9)$, sucrose $(4 \cdot 7)$, xylose $(4 \cdot 0)$. The 
following compounds were not fermented : adonitol, cellobiose, dextrin, dulcitol, glycerol, inositol, inulin, mannitol, mannose, melezitose, rhamnose, sorbitol, starch, trehalose. Unlike organisms 123 and 678, organism 45910 is a powerful pentose-fermenter, although with hexoses, as befits a heterofermentative lactobacillus, it does not yield as low a $\mathrm{pH}$ value in the medium as these other non- or weakly heterofermentative strains. Repetition of the whole series of fermentation tests yielded exactly the same results. The lactic acid produced from glucose was the inactive variety.

Organism 45910 did not hydrolyse hippurate or liquefy gelatin. It produced no haemolysin for sheep red cells.

Pathogenicity. Non-pathogenic to mice, as organism 123.

\section{DISCUSSION}

No claim is made that all the lactobacilli or related Gram-positive rods in the rumens of these young calves have been isolated. We have merely isolated those which grew well in a solid medium suitable for the isolation of typical rumen streptococci. However, we regard it as significant that no Grampositive catalase-negative rods were isolated from the third group of calves which were killed some weeks after the discontinuance of milk in their ration. This probably means that no lactobacilli from milk become really established in the calves' rumen: they are merely organisms of passage, capable sometimes of multiplying greatly for a while in the rumen of a very young calf (e.g. our $1 \mathrm{C}$ aged 32 days), but not persisting for long after their source is withdrawn. It is probably not to the advantage of the calf that lactobacilli should multiply greatly in its rumen for, of all saccharolytic bacteria, the homofermentative lactobacilli most quickly produce a low $\mathrm{pH}$ value and thereby effectively stop the development of 'true' rumen bacteria which flourish best between pH 6 and 7. Our calf $1 \mathrm{C}$, which had a large population of an anaerobic lactobacillus in its rumen, also had a low rumen $\mathrm{pH}$ value of $\mathbf{5 \cdot 0}$.

With regard to the identification of our three catalase-negative rod organisms, the strongly heterofermentative organism 45910 (represented by four isolates from two different calves, one aged 33 and the other 54 days) presents the least difficulty, since its biochemical reactions agree very well with those of Lactobacillus brevis (see group 1 in table 5 of Rogosa et al. 1953). Its ability to hydrolyse aesculin would appear to exclude $L$. fermenti. The only important difference between our organism and the organism of Rogosa et al. is that ours does not hydrolyse hippurate. The obligate anaerobic organism 123 is more difficult to place. It might well pass for a homofermentative lactobacillus unless the test for gas production is very carefully carried out, but it cannot be the well-known homofermentative anaerobe $L$. bifidus, since branching was never observed and it produced laevo- instead of dextrorotatory lactic acid. Its morphology and fermentation reactions agree quite well with the homofermentative L. lactis (see Bergey's Manual, 1948, p. 351) which is said to produce long filaments and threads and to ferment the same sugars as did our organism 123, which also produces laevorotatory lactic acid. Organism 123 might therefore be regarded as an anaerobic variety of $L$. lactis. Our third 
organism (678), although a homofermentative lactic-acid producing rod, cannot be identified with any known organism. It may not belong to the genus Lactobacillus at all since it is motile, ferments mannitol and does not grow at $16^{\circ}$. Although no known species of homofermentative Lactobacillus has these characteristics (see table 1 in Rogosa et al. 1953; Bergey's Manual, 1948), it is clearly difficult to assign organism 678 to any other genus. Until the question of the motility of lactobacilli is cleared up, we hesitate to assign a new species name to this strain. It is clearly very similar in many respects, including fermentation reactions and the kind of lactic acid produced, to the motile lactobacillus isolated by Harrison \& Hansen (1950) from the caecal faeces of turkeys.

Two points of general interest emerge from this study. First, it is evident that the direct morphological approach to rumen microbiology, as advocated by Baker \& Harris (1947-8) and Moir \& Masson (1952), although undeniably useful, has its limitations for it takes no account of the extreme pleomorphism encountered in genera like Lactobacillus, certain members of which, from their biochemical and physiological reactions, might conceivably flourish in the rumen under certain conditions. Our organism 123, for example, consisted in pure culture mostly of filaments, whereas in direct rumen smears only the short rod-forms were in evidence. There can be little doubt, however, that organism 123 did in fact occur in great numbers $\left(c .10^{9} / g\right.$.) in the rumen contents of calf $1 \mathrm{C}$ (see Table 1). Finally, our admittedly limited experience with the fermentation reactions of lactobacilli is more in accord with the findings of Rogosa et al. (1953) who claim reproducibility, than with the conclusions of Briggs (1953), who made no use of these reactions for classificatory purposes on account of their alleged unreliability. Since lactobacilli are carbohydrate fermenters par excellence it surely would be advantageous to use their fermentation reactions for classificatory purposes, as with streptococci, if reproducibility can be assured. The basal medium of Rogosa et al. seems to us to be a definite step forward to this desirable end.

We are grateful to our colleagues: Miss Elizabeth Mackay for the isolation of two anaerobic isolates on the crystal violet medium, and Dr R. J. Pennington for confirming, by the Barker-Summerson method, that our crude fermentation zinc lactates consisted in every case essentially of that salt.

\section{REFERENCES}

Baker, F. \& Harriss, S. T. (1947-8). Microbial digestion in the rumen (and caecum) with special reference to the decomposition of structural cellulose. Nutr. Abstr. Rev. 17, 3.

Barker, S. B. \& Summerson, W. H. (1941). The colorimetric determination of lactic acid in biological material. J. biol. Chem. 138, 535.

Bergey's Manual of Determinative Bacteriology, 6th ed. (1948). Ed. by BrEED, R. S., Murray, E. G. D. \& Hitchens, A. P. London: Baillière, Tindall and Cox.

Briggs, M. (1953). The classification of lactobacilli by means of physiological tests. J. gen. Microbiol. 9, 234.

Harrison, A.P. \& Hansen, P. A. (1950). A motile lactobacillus from the caecal faeces of turkeys. J. Bact. 59, 444. 
Heald, P. J., Krogh, N., Mann, S. O., Appleby, J. C., Masson, F. M. \& Oxford, A. E. (1953). A method for direct viable counts of the facultatively anaerobic microflora in the rumen of a sheep maintained on a hay diet. J. gen. Microbiol. 9, 207.

Mann, S. O., Masson, F. M. \& Oxford, A. E. (1954). Effect of feeding aureomycin to calves upon the establishment of their normal rumen micro-flora and -fauna. Brit. J. Nutr. (in the Press).

MoIr, R. J. \& Masson, M. J. (1952). An illustrated scheme for the microscopic identification of the rumen micro-organisms of sheep. J. Path. Bact. 64, 343.

Rogosa, M., Wiseman, R. F., Mitchell, J. A., Disraely, M. N. \& Beaman, A. J. (1953). Species differentiation of oral lactobacilli from man including descriptions of Lactobacillus salivarius nov.spec. and Lactobacillus cellobiosus nov.spec. J. Bact. 65, 681.

(Received 13 February 1954) 